\title{
Experimental dynamic analysis of nonlinear beams under moving loads
}

\author{
A. Bellino, S. Marchesiello and L. Garibaldi* \\ Dipartimento di Meccanica, Politecnico di Torino, Torino, Itlay
}

\begin{abstract}
It is well known that nonlinear systems, as well as linear time-varying systems, are characterized by non-stationary response signals. In this sense, they both show natural frequencies that are not constant over time; this variation has however different origins: for a time-varying system the mass, and possibly the stiffness distributions, are changing over time, while for a nonlinear system the natural frequencies are amplitude-dependent. An interesting case of time-varying system occurs when analyzing the transit of a train over a railway bridge, easily simulated by the crossing of a moving load over a beam. In this case, the presence of a nonlinearity in the beam behaviour can cause a significant alteration of the modal parameters extracted from the linearized model, such that the contributions of the two effects are no more distinguishable.

For this study, some reinforced concrete beams have been tested in the framework of a vast project: these beams show a clear softening nonlinear behaviour, well detectable when the excitation is produced by a hammer (free response). If the passage of a carriage is considered, moreover, the variation of natural frequencies is always larger than expected because of this softening nonlinearity.

The article first analyzes theoretically the two effects on the natural frequencies of a simply supported beam, then a numerical and an experimental tests are presented. The identification procedure is conducted with a linear algorithm called ST-SSI, which has been demonstrated to be appropriate for the analysis of non-stationary signals, in particular in presence of moving masses. The article shows that the nonlinear contribution can be well estimated by using this linear tool but, on the contrary, when also moving masses are present, it is difficult to separate the nonlinear effects from the time varying ones.
\end{abstract}

Keywords: Moving load, nonlinear behaviour, Short Time Stochastic Subspace Identification, concrete reinforced beams

\section{Introduction}

During the last years, many efforts have been spent in studying both nonlinear and linear time-varying systems, but the two issues have been always analyzed independently. Actually they are not so dissimilar since they both produce non-stationary signals. Moreover, they both have time-varying natural frequencies, caused by the nonlinear dependence of the restoring force on the displacement or velocity (nonlinear system) or by the variation of the state matrices (time-varying systems). In literature, one of the few examples about the study of systems showing nonlinear and time-varying behaviors is an article of Barthels and Wauer [1], who deals with a mechanical device including the two effects. Allison et al. [2], applied the proper orthogonal decomposition to both nonlinear and time-varying systems, in order to look for the different characteristics.

Generally speaking, however, nonlinear systems are identified with specific tools in the case of random excitations, as in [3,4]. On the other side, time-varying systems are usually analyzed with parametric methods [5] or techniques extended from the linear time-invariant case, such as the "frozen technique" [6]. In particular, for the former ones, the typical case studied is the Duffing oscillator [7], but it is impossible to find a theoretical relationship linking the natural frequency, the signal amplitude and the nonlinearity when the polynomial order defining

\footnotetext{
*Corresponding author: L. Garibaldi, Dipartimento di Meccanica, Politecnico di Torino, Corso Duca degli Abruzzi, 24, Torino, Italy. E-mail: luigi.garibaldi@polito.it.
} 
the nonlinearity is larger than 3 [7]. To solve this problem, recently some approximate methods have been proposed $[8,9]$.

The idea behind this article is to connect the usual approaches applied for the time-varying systems (in particular the method called ST-SSI [6]), with a method for the estimation of the nonlinear frequency in a generic system. This procedure allows obtaining the nonlinearity if a free decay response is analyzed. Since the first objective was to study the feasibility of extending the ST-SSI method for nonlinear time-varying systems, then direct approaches for the identification of the nonlinearity, as NSI [3] or CRP [4] have not been used in this paper.

With the knowledge of only one natural frequency and of the signal amplitudes, it is possible to have some information on the nonlinearity. In this paper the identification of the nonlinear term was performed, without any knowledge of the system input, i.e. without any measure of the forcing function, but simply by using the free decay of the system. Sequentially, in the case of a Nonlinear Time-Varying (NTV) system, this estimation can be used to evaluate the nonlinear contribution in the natural frequency extracted. This is the case of the train travelling on a railway bridge, which cannot be a priori considered to behave as a linear system. It can be modelled by means of a simply supported Eulero-Bernoulli beam, with a moving mass passing on it. The presence of the load modifies the state matrices [10], while a polynomial term can be added to each modal equation in order to simulate a NTV system.

The experimental example considered concerns some concrete reinforced beams tested in Pescara, within a project financed by the Italian Ministry of Research. The beams show a clear softening behaviour, which is estimated by means of the proposed method. Successively, the cases with a moving carriage over the beams are treated.

The results show that the method is able to well estimate the nonlinear effect and to quantify the diminution of the frequency values due to the nonlinearity; the results are verified and tested both for the numerical and for the experimental example.

\section{The method for the nonlinearity estimation}

In this section, firstly an overview over the extraction of the frequency in a general dynamic system is proposed. Since the relationship extracted links the frequency, the signal amplitudes and the nonlinearity, a method for the extraction of the linear and nonlinear stiffness contributions from the identified natural frequencies is successively presented.

The process must be applied on a time-invariant system, and in particular on a free decay response. Finally, the nonlinear behavior just estimated can be used to compare the natural frequency extracted from the time-varying case with the theoretical natural frequency depending only on the moving mass.

\subsection{Mathematical background}

A general single d.o.f. nonlinear oscillator can be represented in the form [9]

$$
u^{\prime \prime}+f(u)=0
$$

Let us consider the initial conditions expressed by $u(0)=A$ and $u^{\prime}(0)=0$. Since there is no dissipation, the total energy is constant, therefore $1 / 2\left(u^{\prime}\right)^{2}+F(u)=F(A)$, where

$$
F(u)=\int f(u) d u
$$

By assuming that the initial approximate guess has the form $u(t)=A \cos (\omega t)$, then it is simple to write:

$$
\frac{\omega^{2} A^{2}}{2} \sin ^{2} \omega t+F(A \cos \omega t)-F(A)=0
$$

A good approximation of the angular frequency [9] is given by 


$$
\omega=\sqrt{\frac{2(F(A)-F(A \cos \omega t))}{A^{2} \sin ^{2} \omega t}}
$$

evaluated for $\omega t=\pi / 4$. Now, let's consider a dynamic system with small damping, such that the hypothesis of energy conservation can be considered valid. The nonlinear function $f(x)$ can be written as sum of linear and nonlinear stiffness contributions:

$$
f(x)=\frac{1}{m} \sum_{i=1}^{N} k_{i} x^{i}
$$

where $Q_{L}(x)=k_{1} x$ is the linear force and $Q_{N L}(x)=\sum_{i=2}^{N} k_{i} x^{i}$ is the nonlinear one. By integrating Eq. (5),

$$
F(x)=\frac{1}{m} \sum_{i=1}^{N} \frac{k_{i}}{i+1} x^{i+1}
$$

Collocation in $\omega t=\pi / 4$ gives:

$$
\omega=\sqrt{\frac{4}{m A^{2}}\left(\sum_{i=1}^{N} \frac{k_{i}}{i+1} A^{i+1}-\sum_{i=1}^{N} \frac{k_{i}}{i+1} A^{i+1} 2^{-\frac{i+1}{2}}\right)}=\sqrt{\frac{1}{m}\left(\sum_{i=1}^{N} g_{i} k_{i} A^{i-1}\right)}
$$

where the coefficients $g_{i}$ are given by

$$
g_{i}=\left(\frac{4-2^{\frac{3-i}{2}}}{i+1}\right)
$$

The maximum value $\bar{g}_{i}=1$ is reached for $i=1$ (linear case), where $\omega=\sqrt{k_{1} / m}$. In Eq. (7) a direct connection between the nonlinear stiffnesses and the angular frequency is obtained, similar to Eq. (5) but scaled with the known coefficients $g_{i}$. The square of the natural frequency $f=\omega / 2 \pi$ can be therefore written as:

$$
f^{2}=\frac{1}{4 \pi^{2} m} \sum_{i=1}^{N} g_{i} k_{i} A^{i-1}
$$

\subsection{Time-varying frequencies}

In order to completely characterize the nonlinearity present in a generic system, all the different coefficients $k_{i}$ must be identified. The first step of the procedure is the identification of the time-varying natural frequencies by means of the ST-SSI method [6], which is able to obtain a value of frequency for all the time instants, by applying the classical subspace methods in successive signal windows. This method is based on Van Overschee's work but it is applied to time-varying systems. The main idea is to consider the matrices constant during each time step and, under this assumption, to apply all the results concerning the time-invariant systems. This procedure is often called frozen technique.

The state-space model, for a Linear Time-Varying (LTV) system, can be written as

$$
\left\{\begin{array}{l}
x(k+1)=A(k) x(k)+B(k) u(k)+w(k) \\
y(k)=C(k) x(k)+D(k) u(k)+v(k)
\end{array}\right.
$$


The whole time period is divided in many windows, in which the system is considered time-invariant. The windows are chosen superimposed and shifted by the sampling period.

The modal parameters are directly extracted from the matrix $A(k)$ and they are called respectively pseudo-damping factors and pseudo-damped natural frequencies $[11,12]$. For simplicity, they are usually renamed as damping factors and natural frequency, as in the linear time-invariant case.

\subsection{Extraction of the nonlinear stiffness contributions}

Since the present method is built for a single d.o.f. system, then only one frequency (typically the first) is taken into account, and hence the considered mass is the corresponding modal mass. Once extracted the frequency, let's consider the square of the natural frequency just extracted and perform a $(N-1)$-order polynomial regression such that

$$
4 \pi^{2} m \sum_{i=1}^{N} p_{i} A^{i-1}=\sum_{i=1}^{N} g_{i} k_{i} A^{i-1}
$$

where $p_{i}$ are the regression coefficients. The parameter $N$ must be chosen to obtain a good representation of $f^{2}$. The stiffness coefficients can be easily found through the following relationship:

$$
\tilde{k}_{i}=4 \pi^{2} m \frac{p_{i}}{g_{i}}
$$

Since the method is built by considering a relationship among the amplitude, the frequency and the nonlinearity, the optimal case it is adapted for is the free decay time-invariant response. As a matter of example, for a train crossing a bridge, this situation corresponds to the time section when the train leaves the bridge, which hence vibrates without any external force acting on.

\section{Modelling of a nonlinear beam with a moving load}

This section deals with the modeling and the simulation of a simply supported nonlinear beam with a moving load travelling on it. Once formalized the equation of motion, a numerical case is analyzed.

\subsection{Model of a simply supported beam travelled by a moving load}

The equation of motion of an Eulero-Bernoulli beam, travelled by a moving load is

$$
E I \frac{\partial^{4} w}{\partial x^{4}}+b \frac{\partial w}{\partial t}+\mu \frac{\partial^{2} w}{\partial t^{2}}=r(t) \delta(x-v t)
$$

where, $w$ is the vertical displacement of the beam, $E I$ is the bending stiffness, $b$ is the coefficient of external viscous damping, $\mu$ is the mass per unit of length, $v$ is the load velocity and the interaction force $r(t)$ is

$$
r(t)=m g-m \ddot{u}
$$

where $m$ is the load mass, $g$ is the gravity acceleration and $\ddot{u}$ the acceleration at the interface between the beam and the load. Since $u(t)=w(\xi(t), t)=\sum_{i} \phi_{i}(\xi(t)) y_{i}(t)=\boldsymbol{\varphi}(\xi(t))^{T} \mathbf{y}(t)$, where $\phi_{i}$ denotes the orthonormalized mode shape, $\xi(t)=v t$ is the load position and $y_{i}$ the generalized displacements, consequently 


$$
\ddot{u}(t)=v^{2} \varphi^{I I}(\xi(t))^{T} \mathbf{y}(t)+2 v \varphi^{I}(\xi(t))^{T} \dot{\mathbf{y}}(t)+\varphi(\xi(t))^{T} \ddot{\mathbf{y}}(t)
$$

By using the first mode approximation it is possible to write the equation of motion, according to the procedure described in [10]. Then, in order to take into account some nonlinear effects, a nonlinear polynomial term has been simply added to the first member of the equation, as for the time-invariant cases discussed by [13]:

$$
\begin{aligned}
& M(t) \ddot{y}(t)+C(t) \dot{y}(t)+K(t) y(t)+\sum_{i=2}^{N} h_{i} y^{i}(t)=T(t) \\
& M(t)=1+m \varphi(\xi(t)) X(t) \varphi(\xi(t))^{T} \\
& C(t)=c_{0}+2 m v \varphi(\xi(t)) X(t) \varphi^{I}(\xi(t))^{T} \\
& K(t)=\omega^{2}+m v^{2} \varphi(\xi(t)) X(t) \varphi^{I I}(\xi(t))^{T} \\
& T(t)=m g \varphi(\xi(t)) X(t)
\end{aligned}
$$

where $X(t)$ is a window function, equal to 1 if the carriage is on the beam and equal to 0 otherwise, $h_{i}$ denote the normalizations of the $N$ nonlinear stiffness coefficients $k_{i}$, and $c_{0}$ is the normalized damping.

\subsection{A numerical example}

Let's consider Eq. (16) for the beams with the characteristics listed in Table 1, where a cubic softening nonlinearity has been inserted. Eq. (16) was numerically integrated and accelerations of 4 points along the structure were corrupted by $3 \%$ of white noise.

In Fig. 1a, the displacements of the middle point of the beam are presented. In Fig. 1b, the first natural frequency extracted by means of the identification method is shown, together with the reconstructed one. The frequency is directly connected with the displacement level: at the beginning, the displacement is maximum and the frequency is minimum, then while the first quantity decreases, the second quantity increases. This is a typical behaviour of a softening system. In Fig. 2, the nonlinear stiffness force estimated is compared with the correct one. It has been obtained by

$$
f_{R E C}=\frac{1}{2 \pi} \sqrt{\frac{1}{m}\left(\sum_{i=1}^{N} g_{i} \tilde{k}_{i} A^{i-1}\right)}
$$

Both the images confirm that the results are very similar to the right values. Successively, a moving load with mass $m_{C}=1 \mathrm{~kg}$ (equal to around $8 \%$ of the beam mass) and constant velocity $v_{C}=0.1 \mathrm{~m} / \mathrm{s}$ is chosen. An initial impulse is inserted in order to simulate an irregularity at the beginning of the load transit.

Table 1

Characteristics of the beam considered

\begin{tabular}{lll}
\hline \multicolumn{1}{c}{ Young's modulus } & $E$ & \multicolumn{1}{c}{$70 \mathrm{GPa}$} \\
\hline Mass per unit of length & $\mu$ & $6.71 \mathrm{~kg} / \mathrm{m}$ \\
Beam length & $L$ & $1.86 \mathrm{~m}$ \\
Nonlinearity & $k_{3}$ & $-210^{6} \mathrm{~N} / \mathrm{m}^{3}$ \\
Normalized damping & $c_{0}$ & $1.87 \mathrm{~kg} / \mathrm{m} / \mathrm{s}$ \\
\hline
\end{tabular}


(a)

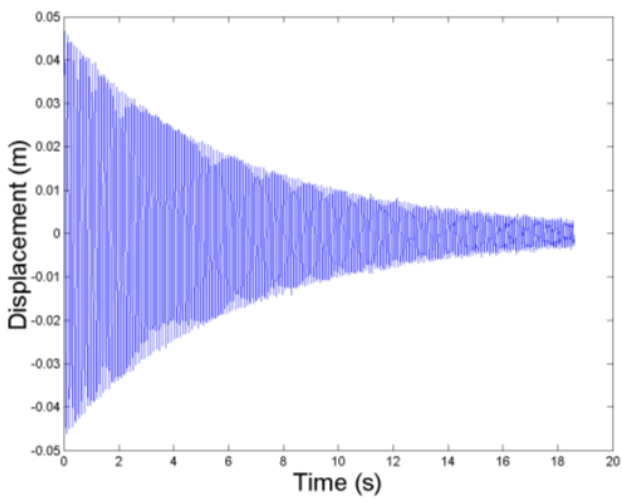

(b)

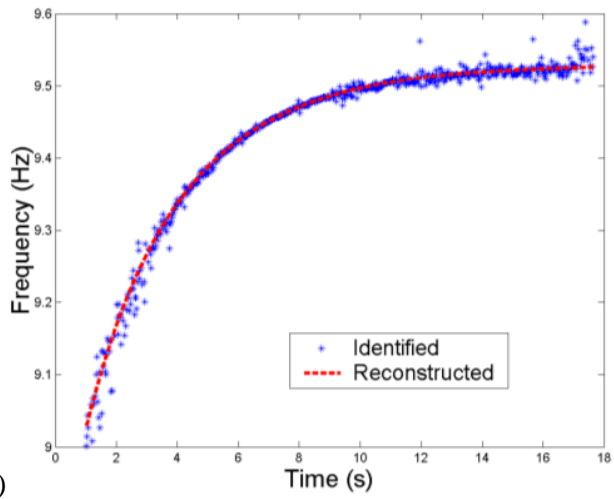

Fig. 1. Displacements at the middle point of the beam (a) and reconstructed first natural frequency with that identified from the ST-SSI method (b).

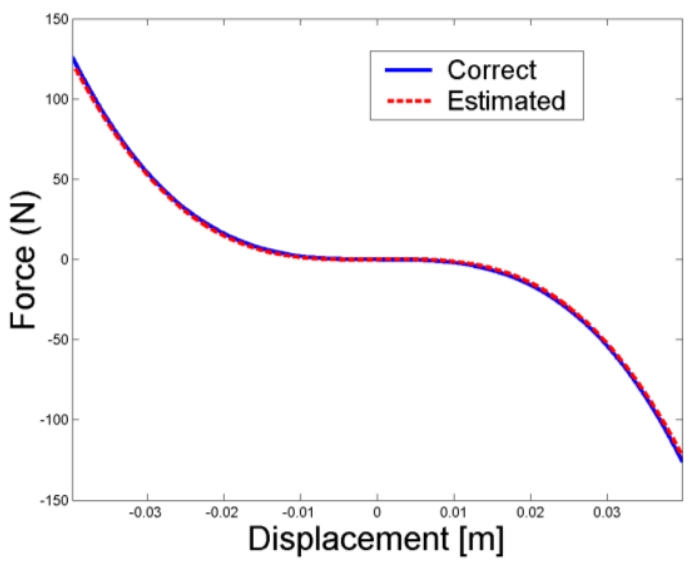

Fig. 2. Nonlinear stiffness force contribution estimated compared with the correct one.

(a)

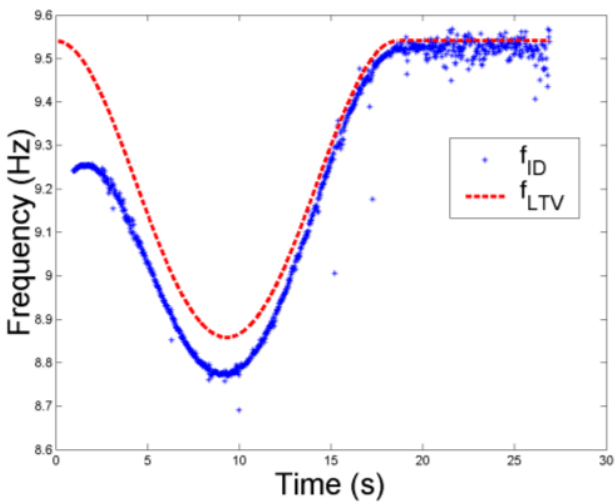

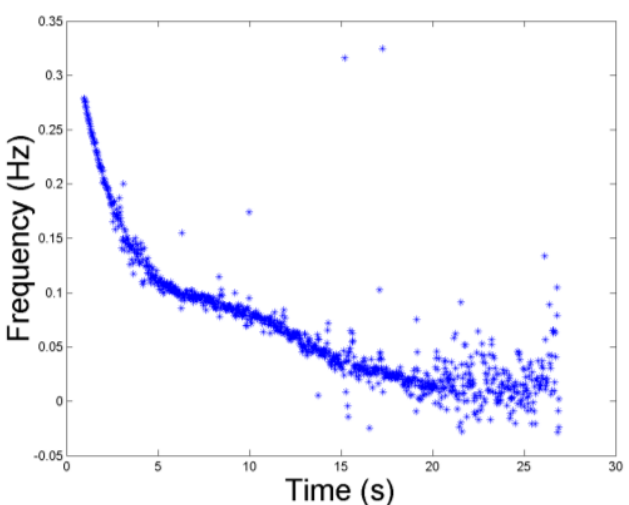

(b)

Time (s)

Fig. 3. First natural frequency extracted with the identification method compared with the theoretical natural frequency depending only on the moving mass (a) and difference between the two curves (b).

In Fig. 3a, the natural frequency identified by means of the method ST-SSI is compared with the theoretical frequency depending only on the moving mass, which is written as follows: 


$$
f_{L T V}=\frac{f_{L T I}}{\sqrt{1+m \varphi(\xi(t))^{2}}}
$$

where $f_{L T I}$ is the beam frequency, obtained from $\tilde{k}_{1}$ :

$$
f_{L T I}=\frac{1}{2 \pi} \sqrt{\frac{\tilde{k}_{1}}{m_{r}}}
$$

where $m_{r}$ is the modal mass of the beam, which is equal to half the total mass, for a simply supported homogeneous Euler-Bernoulli beam.

In Fig. $3 b$ the differences between the two curves are represented. In the first part of the transit, the difference is very large because of the impact force at the beginning of the time history. Afterwards, the difference decreases because the signal level is lower and the unique force acting on the system is that induced by the moving load. The right zone is relative to the free decay of the beam, since the moving load is no more present on it. The estimation of the frequency in that zone is not perfect because of the signal level which is very low, but it is possible to see that the frequency extracted is slightly increasing, due to the nonlinear softening effect.

Conclusively, the direct use of a linear analysis (like the identification method proposed) on time-varying systems is not applicable in presence of nonlinear effects, but it is necessary to first consider a free-decay (without time-variability) in order to assess the nonlinearity.

\section{Pescara beams}

Within a huge project titled "Monitoring and diagnostics of railway bridges by means of the analysis of the dynamic response due to train crossing", financed by Italian Ministry of Research, many experimental tests on several reinforced concrete beams have been performed in Pescara, Italy. In this section, firstly a description of the beams and the tests is presented, then the results of the method are discussed.

\subsection{Beam characteristics}

In Table 2, the beam characteristics are summarized. These values can be considered as a general reference for all the beams analyzed, bearing in mind that the beams show slightly different values one each other. The sampling frequency of accelerations acquisition is equal to $4096 \mathrm{~Hz}$.

Two different types of tests have been conducted:

- Release test: the beam has been lifted a few centimeters by one end and then it has been released, such that it bumped against the support, and the response was a free decay, therefore this test is useful for the identification of the nonlinearity.

- Load transit test: a moving load (with mass equal to $11.37 \mathrm{~kg}$ ) has been placed on the beam and pulled by means of a rope. Two more beams have been added to the system as acceleration and deceleration ramps, respectively, as it can be seen in Fig. 3a.

Table 2

Characteristic of the beam

\begin{tabular}{ll}
\hline Total length & $l=3.2 \mathrm{~m}$ \\
Length between the supports & $L=3 \mathrm{~m}$ \\
Area & $A=0.0072 \mathrm{~m}^{2}$ \\
Moment of inertia & $I=4.32 \cdot 10^{-6} \mathrm{~m}^{4}$ \\
Young modulus & $E=50.481 \mathrm{GPa}$ \\
Density & $\rho=2597.2 \mathrm{Kg} / \mathrm{m}^{3}$ \\
\hline
\end{tabular}


(a)

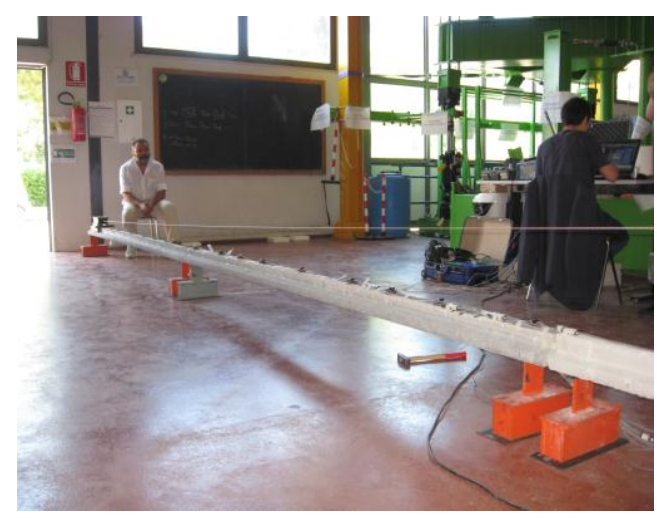

(b)

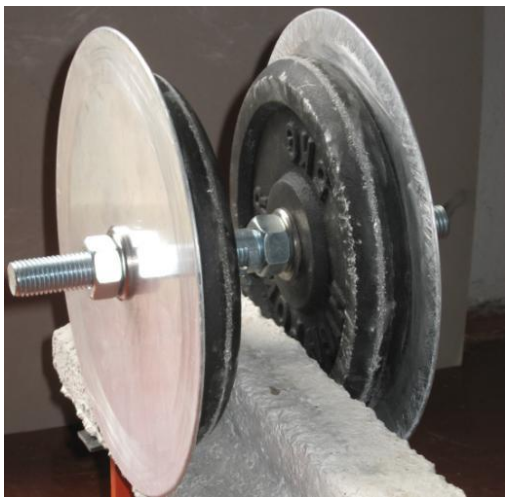

Fig. 4. The configuration test (a) and the mass crossing the beam (b).

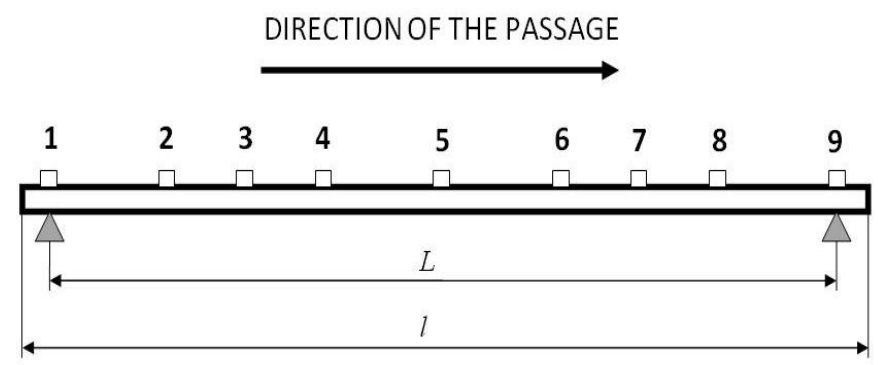

Fig. 5. The scheme of the beams analyzed in Pescara (b).

In Fig. 4a, the three consecutive beams and the experimental set-up are presented, while in Fig. $4 \mathrm{~b}$ the moving mass is depicted. In Fig. 5, a simplified scheme of the beams is shown. It is possible to note that the supports are not exactly at the ends of each beam.

\subsection{Results of the method}

In this experimental case, there are two main objectives:

1. Estimate the nonlinear contribution only

2. Show how the nonlinearity influences the first natural frequency of the beam, by comparing the frequency identified with the theoretical one (with only moving mass effects).

The nonlinear effect is identified by analyzing the time history relative to the release case. The first natural frequency and the first damping factors are estimated with the ST-SSI method and they are depicted in Fig. 6. The natural frequency is monotonically increasing, and the asymptote is the frequency of the underlying linear system, equal to $19.06 \mathrm{~Hz}$, obtained by using Eq. (23). The damping factor, contrarily, is monotonically decreasing. This behavior is typical of a nonlinear softening system.

An integration process is used to extract the displacement values, from the recorded accelerations. For simplicity, only the accelerometer number 5 has been considered for the application of the procedure.

In Fig. 7, the displacement peaks are depicted (a), together with the estimation of the nonlinear force (b), after having chosen $N=6$. In Fig. 7a, the trend is typical of a free damped decay, while in Fig. $7 \mathrm{~b}$ the nonlinear force is qualitatively similar to the numerical case, because this is a softening system. 

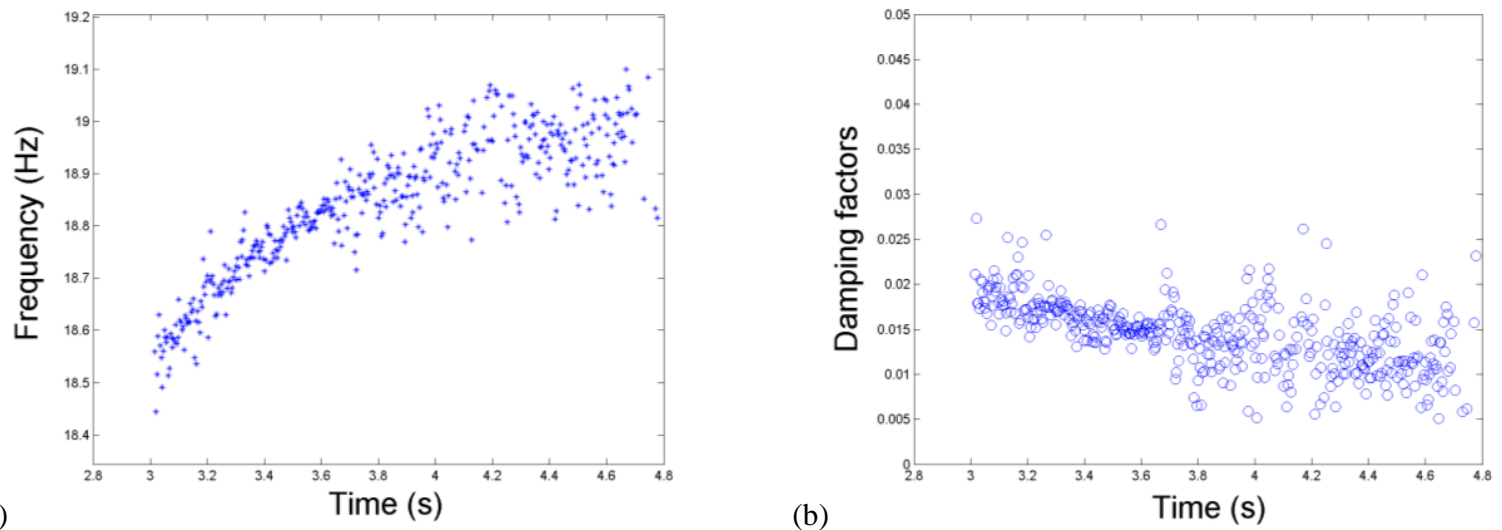

Fig. 6. Identified natural frequency (a) and damping factor (b), along the free decay of the release test.

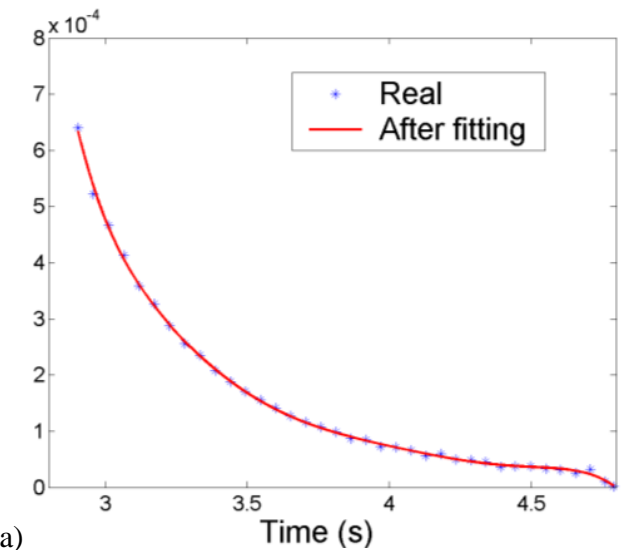

(b)

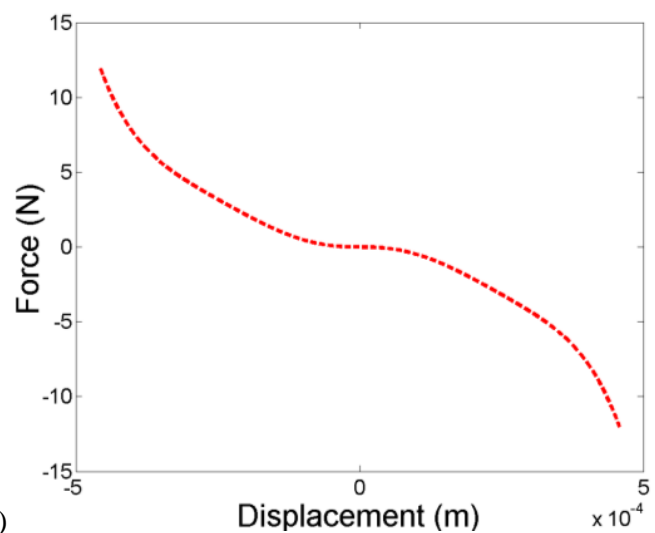

Fig. 7. Peaks of the displacement (a) and nonlinear force estimated (b), release test.
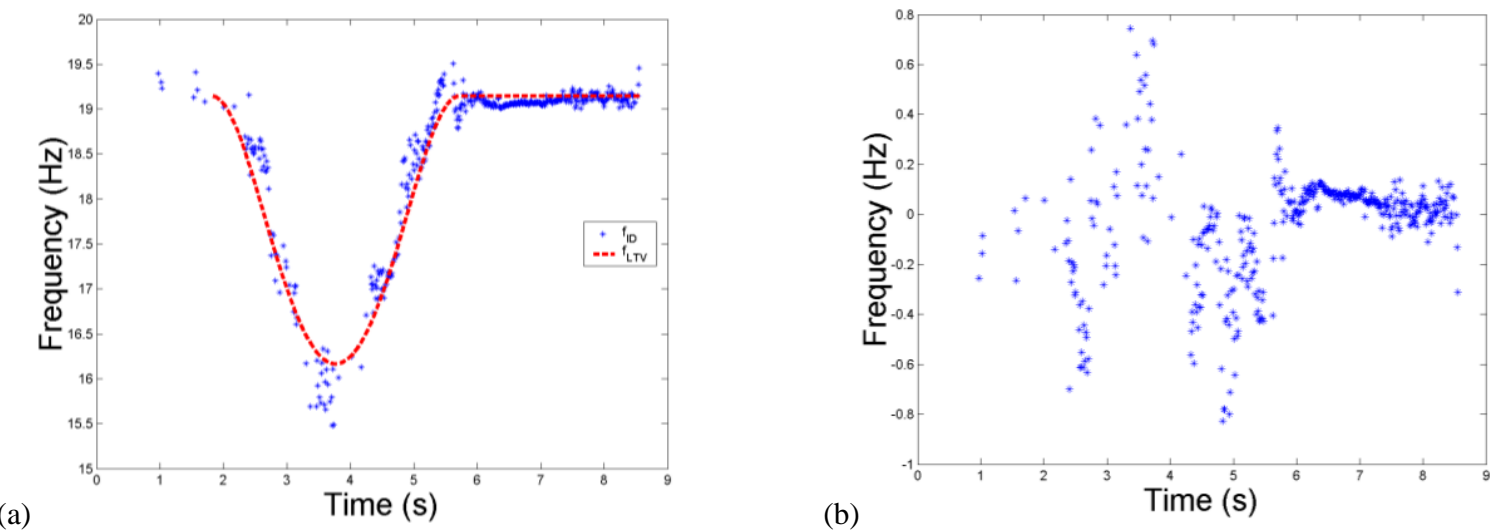

Fig. 8. First natural frequency extracted by means of ST-SSI compared with the theoretical natural frequency depending only on the moving mass (a) and difference between the two curves (b).

In Fig. 8a, the identified frequency is depicted, together with the theoretical one, depending only on the moving load, while in Fig. $8 \mathrm{~b}$ the difference between the two curves is visualized. The theoretical frequency has been obtained by considering the modal mass as half the total mass and a constant velocity, according to Eq. (22). 
Actually, since the velocity is not constant, there are some small variations respect to the theoretical case. However, the values extracted near the beam centre are smaller respect to the theoretical curve and this fact agrees with the presence of the softening nonlinearity. Moreover, at the end of the transit, the frequency increases in the same way as in Fig. 6a, towards the beam frequency.

As for the numerical case, the extracted frequency of the case containing both the nonlinearity and the time-variability gives a qualitative indication of the presence of the two effects, but the linear tool ST-SSI does not help in separating them.

\section{Conclusions}

In the paper, different issues have been treated, in particular the estimation of the nonlinear restoring force in a nonlinear system and the nonlinear effects on the natural frequencies in a NTV system. The whole procedure has been applied both to a numerical case of a simply supported nonlinear beam and to an experimental case of a pre-stressed beam showing a softening nonlinear behaviour. This procedure requires different steps but the computational time is negligible. The results obtained on both the numerical and the experimental tests clearly show the importance of the nonlinear contribution on the dynamic response.

As highlighted in the numerical and in the experimental example, when dealing with nonlinear time-varying systems, it is advisable to first consider a free-decay response, in which the nonlinear contribution can be estimated. This operation is executed because the linear identification tool considered is not able to treat both the nonlinear and time-varying effects simultaneously.

\section{References}

[1] P. Barthels and J. Wauer, Non-smooth and time-varying systems of geometrically nonlinear beams, Journal of Sound and Vibration $\mathbf{3 1 5}$ (2008), 455-466.

[2] C.A. Allison, A.K. Miller and D.J. Inman, A time-varying identification method for mixed response measurements, Journal of Sound and Vibration 319 (2009), 850-868.

[3] S. Marchesiello and L. Garibaldi, A time domain approach for identifying nonlinear vibrating structures by subspace methods, Mechanical System and Signal Processing 22 (2008), 81-101.

[4] C.M. Richards and R. Singh, Identification of Multi-degree-of-freedom non-linear systems under random excitations by the reverse path spectral method, Journal of Sound and Vibration 13 (1998), 673-708.

[5] A.G.D. Poulimenos and S. Fassois, Parametric time-domain methods for non-stationary random vibration modelling and analysis - A critical survey and comparison, Mechanical System and Signal Processing, 20, 2006, pp. 763-816.

[6] A. Bellino, L. Garibaldi and S. Marchesiello, Time-Varying Output-Only Identification of a cracked beam, Key Engineering Materials 413-414 (2009), 643-650.

[7] A. Beléndez, G. Bernabeu, J. Francés, D.I. Méndez and S. Marini, An accurate closed-form approximate solution for the quintic Duffing oscillator equation, Mathematical and Computer Modelling 52 (2010), 637-641.

[8] S.S. Ganji, D.D. Ganji, M.G. Sfahani and S. Karimpour, Application of AFF and HPM to the systems of strongly nonlinear oscillation, Current Applied Physics 10 (2010), 1317-1325.

[9] I. Mehdipour, D.D. Ganji and M. Mozaffari, Application of the energy balance method to nonlinear vibrating equations, Current Applied Physiscs 10 (2010), 104-112.

[10] B. Biondi and G. Muscolino, New improved series expansion for solving the moving oscillator problem, Journal of Sound and Vibration 281 (2005), 99-117.

[11] K. Liu, Identification of linear time-varying systems, Journal of Sound and Vibration 206 (1997), 487-505.

[12] K. Liu, Extension of modal analysis to linear time-varying systems, Journal of Sound and Vibration 226 (1999), $149-167$.

[13] M.F. Platten, J.R. Wright, G. Dimitriadis and J.E. Cooper, Identification of multi-degree of freedom non-linear systems using an extended modal space model, Mechanical System and Signal Processing 23 (2009), 8-29. 

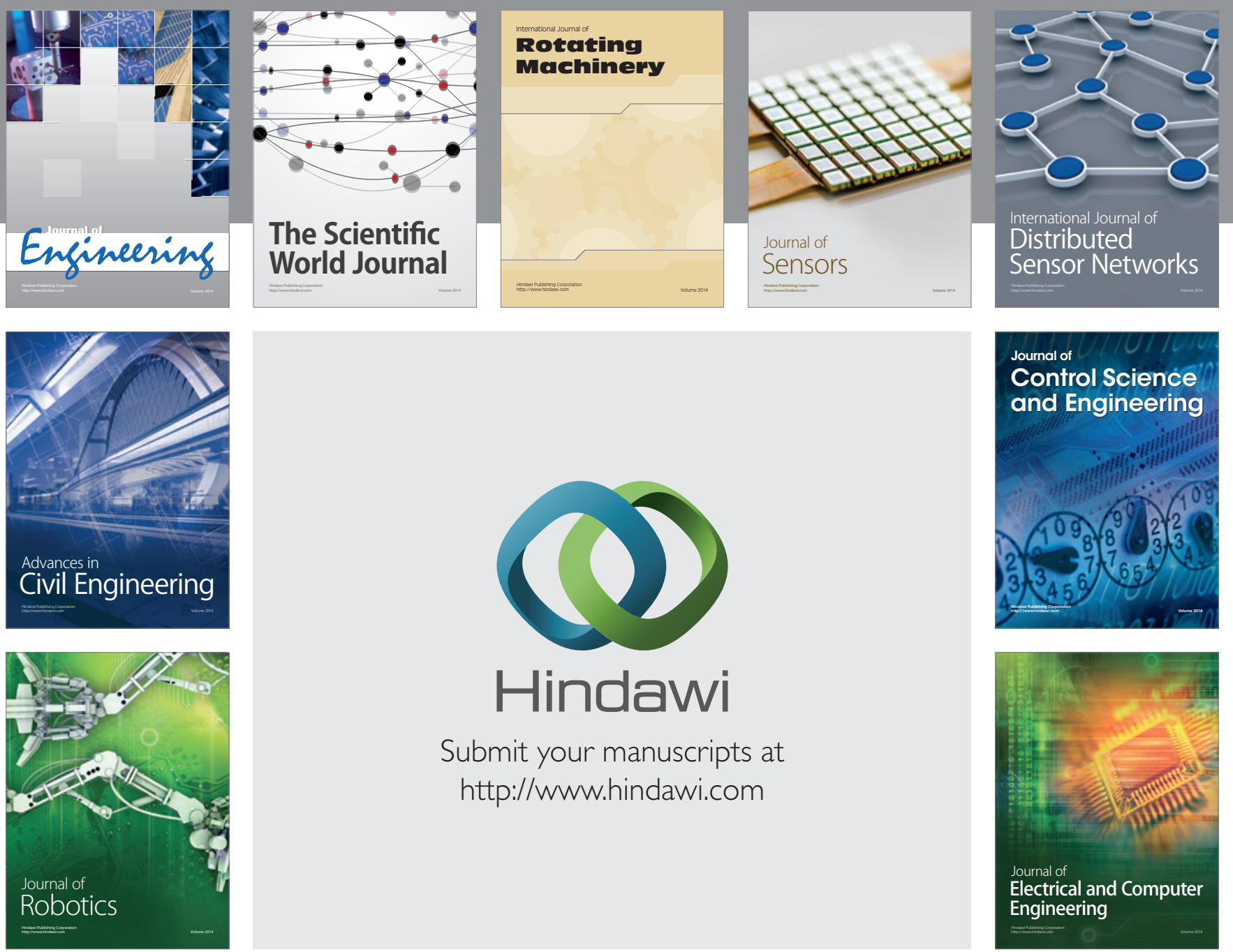

Submit your manuscripts at

http://www.hindawi.com
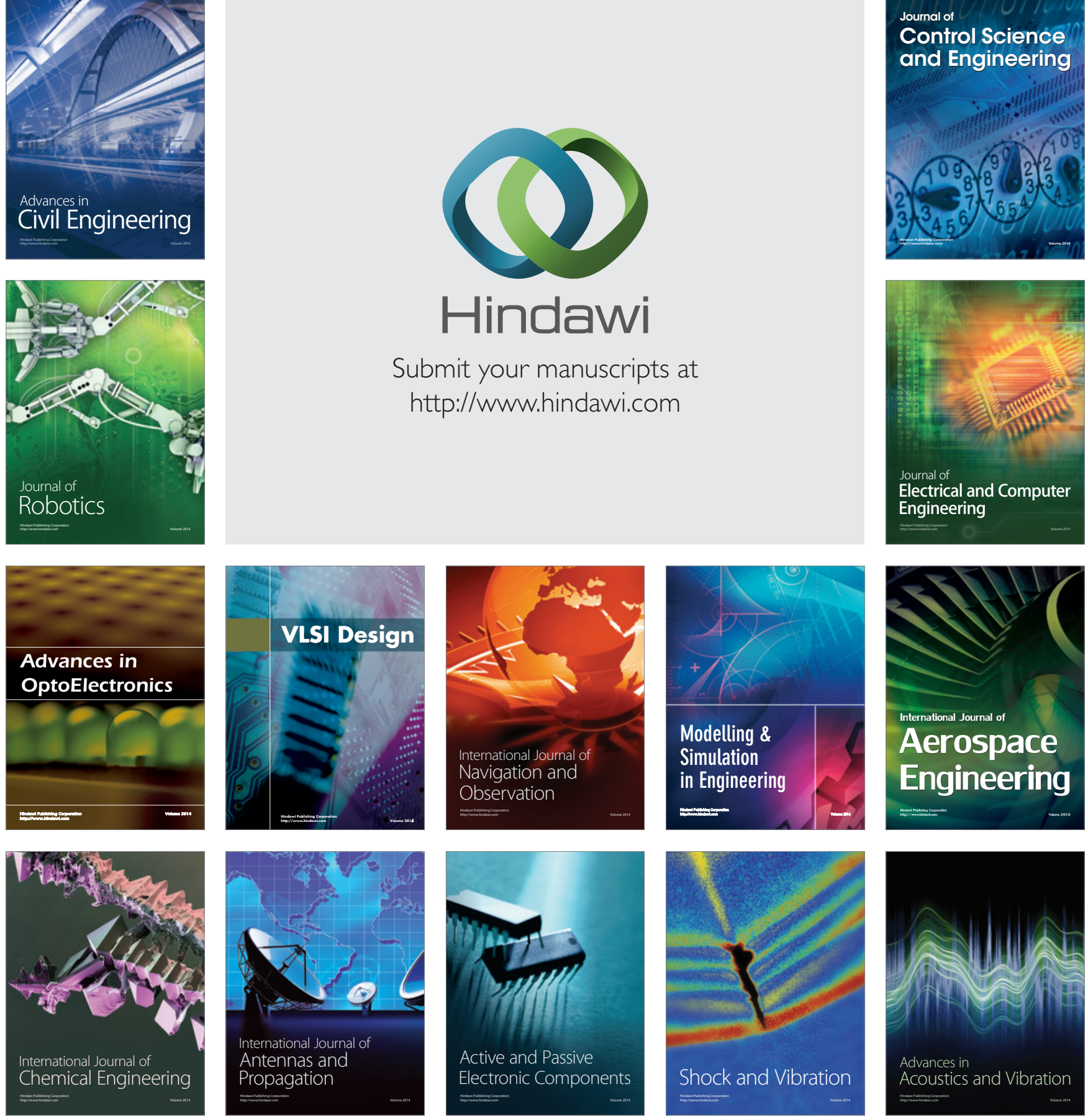